\title{
Características epidemiológicas e clínicas dos casos notificados da tuberculose na cidade de Tianguá, Ceará, Brasil (2008-2013)
}

\author{
Epidemiological and clinical characteristics of reported cases \\ of tuberculosis in Tianguá city, Ceará, Brazil (2008-2013)
}

Recebido em: 08/09/2015 Aceito em: $\quad 30 / 05 / 2016$
Germana Fontenele SANTOS ${ }^{1}$; Rafael Gonzalez FROTA² Carlos Eduardo Barros MORAES ${ }^{2}$; Maria Auxiliadora Silva OLIVEIRA ${ }^{2,3}$

${ }^{1}$ Santa Casa de Misericórdia de Sobral. Rua Antonio Crisóstomo de Melo 919, Centro. Sobral-CE, CEP: 62010-550, Brasil; ${ }^{2}$ Faculdade de Medicina, Instituto Superior de Teologia Aplicada - INTA. Rua Coronel Antonio Rodrigues Magalhães, Bairro Dom Expedito Lopes, 359, Sobral, CE, CEP: 62010-550, Brasil; ${ }^{3}$ Universidade Federal do Ceará - UFC. Av. Comandante Maurocélio Rocha Ponte, 100, Derby - CE, CEP 62042-280, Brasil. E-mail:ecobio@zipmail.com.br

\section{ABSTRACT}

This study aimed to describe the clinical and epidemiological profiles of reported cases of tuberculosis in the city of Tianguá, CE. This is a retrospective, descriptive study was quantitative, and exploratory, with document analysis taking as an information source tuberculosis cases reported in the Notifiable Diseases Information System (SINAN) reported between the years 2008 and 2013. The variables analyzed were: gender, education, age, type of entry, clinical form and co-infection and HIV. It was recorded 98 cases of tuberculosis to the Municipal Secretary of Health of the municipality. Of these, $65.3 \%$ are male. The age group most affected by the disease was $40-59$ years, with $40.8 \%$. As to education, individuals with up to primary education were predominant. The illiteracy rate was $16.4 \%$. There was predominance of the pulmonary form with $85.7 \%$. According to the distribution of cases according to tuberculosis co-infection - HIV, there was a prevalence of individuals with tuberculosis HIV-uninfected.

Keywords: tuberculosis; Information System for Disease Notification; infectious disease

\section{RESUMO}

O presente trabalho teve como objetivo descrever as características clínico-epidemiológicas dos casos notificados da tuberculose na cidade de Tianguá, CE. Trata-se de um estudo retrospectivo, descritivo, do tipo quantitativo, sendo exploratório com análise documental, tomando como fonte de informação os casos de tuberculose notificados no Sistema de Informação de Agravos de Notificação (SINAN) notificados entre os anos 2008 e 2013. As variáveis analisadas foram: gênero, escolaridade, faixa etária, tipo de entrada, forma clínica e co-infecção e HIV. Foram registrados 98 casos de tuberculose à Secretaria Municipal de Saúde do município. Desses, 65,3\% são do gênero masculino. A faixa etária mais acometida pela doença foi a de 40 a 59 anos com 40,8\%. Com relação à escolaridade, foram predominantes os indivíduos com até Ensino Fundamental. O índice de analfabetismo foi de 16,4\%. Foi verificada uma predominância de $85,7 \%$ da forma pulmonar. De acordo com a distribuição dos casos, segundo coinfecção tuberculose-HIV, houve prevalência de indivíduos com tuberculose não infectados pelo HIV.

Palavras-chave: tuberculose; Sistema de Informação e Notificação de Doenças; doenças infecciosas 


\section{INTRODUÇÃO}

A tuberculose (TB) é uma antiga doença infecciosa, que nos dias atuais, tornou-se uma grande preocupação mundial. Essa doença, provocada pela bactéria Mycobacterium tuberculosis, foi isolada em 1882 pelo cientista alemão Robert Koch e é basicamente transmitida pelo ar, podendo atingir diferentes áreas do corpo (1)

Esse agravo voltou a ocupar um papel de destaque entre as principais doenças infecto-contagiosas, especialmente em países em desenvolvimento. Isto se deve principalmente a dois fatores: $\mathrm{o}$ aparecimento cada vez frequente de cepas de bacilos resistentes aos fármacos conhecidos e o surgimento, em 1980, da Síndrome de Imunodeficiência Adquirida (AIDS). É importante mencionar outros fatores que são responsáveis para 0 aumento global da TB tais como aumento da pobreza, desordem social e moradias superlotadas em países em desenvolvimento, falta de informação, ausência de investimentos e a falta programas eficazes no controle da TB (1)

A população mundial gira em torno de 7 bilhões de habitantes. Destes, a Organização Mundial de Saúde (OMS) estima que um terço seja infectado pelo bacilo da TB. As estimativas demonstram que em 2010 ocorreram 8,8 milhões de novos casos e 1,45 milhões de óbitos pela doença. Mais de 500 mil casos da TB são multirresistentes e 700 mil são coinfectados com o HIV/AIDS (3).

O Brasil está entre os 22 países que concentram $82 \%$ dos casos de TB no mundo. A TB foi responsável por 4,8 mil óbitos, 71 mil novos casos, e é a terceira causa de mortes por doenças infecciosas e a primeira de óbitos de pacientes que viviam com HIV/AIDS. Anualmente surgem no Brasil, aproximadamente, 111 mil novos casos (4).

A tuberculose constitui prioridade de saúde pública no Brasil, caracterizando-se por apresentar elevada incidência e distribuição espacial heterogênea nas diferentes regiões do país, guardando estreita relação com as condições sócioeconômicas da população (2).

O presente trabalho teve como objetivo traçar o perfil das notificações da tuberculose, em relação às características epidemiológicas e clínicas, no município de Tianguá, Ceará, no período de 2008 a 2013.

\section{MATERIAIS E MÉTODOS}

Essa pesquisa é descritiva e retrospectiva, de análise quantitativa e condensação dos dados por meio de estatística descritiva, tomando como fonte de informação os casos de tuberculose notificados no Sistema de Informação de Agravos de Notificação (SINAN) entre os anos 2008 e 2013. Os dados foram coletados diretamente das fichas de notificação/investigação de cada paciente. Para uma coleta homogênea, foi elaborado um instrumento baseado nos dados das fichas de notificações/investigações. Foram analisadas 98 fichas.

Foram incluídas todas as notificações no período de 2008 a 2013. As variáveis analisadas foram: gênero, escolaridade, faixa etária, tipo de entrada, forma clínica e coinfecção e HIV.

Foi utilizado o programa SPSS - Statistical Package of The Social Sciences (versão 15.0 for Windows), com a utilização estatística descritiva (frequências e proporções) para as variáveis categóricas.

A pesquisa foi cadastrada na Plataforma Brasil e submetida ao Núcleo de Ensino e Pesquisa de Sobral (NEPS) /Comitê de Ética em Pesquisas envolvendo Seres Humanos (CEP) da Universidade Estadual Vale do Acaraú - UVA, sendo aprovada com parecer de número 889.716. Por motivos superiores foi solicitado dispensa de TCLE, tendo sido aceito pelo referido comitê.

\section{RESULTADOS E DISCUSSÃO}

A primeira ação do referente trabalho foi analisar as notificações de tuberculose; e após essa análise, foi observado que durante o período de 2008 a 2013, foram registrados 98 casos de tuberculose à Secretaria Municipal de Saúde de Tianguá, CE. A distribuição dos 98 casos de tuberculose conforme o ano de notificação, evidenciou um pico de incidência de tuberculose no ano de 2008, com 23 casos registrados (Tabela 1).

É possível perceber que houve diminuição do número de casos notificados, chegando a $11 \mathrm{em} \mathrm{2013,}$ uma taxa de crescimento negativo de $52,17 \%$ em relação ao ano de 2008. Essa redução está dentro do esperado pelo Plano Global para o Combate à TB 2011-2015 ("STOP TB"), proposto pela OMS, uma estratégia que pode ser entendida como um conjunto de boas práticas para o controle da $\mathrm{TB}$, tendo como objetivo reduzir pela metade a carga da doença até 2015 , tanto a taxa de incidência quanto a taxa de mortalidade (em comparação com os valores de 1990) como também eliminar a TB como problema de saúde pública até 2050 (5).

Também há que se levar em consideração que essa redução pode estar associada não exatamente à redução dos casos de pessoas infectadas no município, mas 


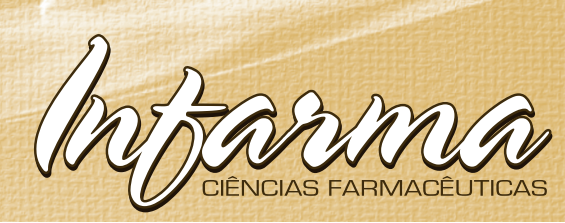

também a possíveis subdeteç̧ões ou subnotificações de novos casos. O próprio SINAN apresenta limitações que dificultam o conhecimento real da doença, como a subnotificação, a baixa completude dos dados e a precariedade das informações relacionadas ao encerramento dos casos de TB (6).
O acometimento de pacientes do gênero masculino foi significativamente maior que os pacientes do gênero feminino. Pode ser observado, na Tabela 2, que, das 98 notificações analisadas, o gênero masculino prevaleceu com $65,3 \%$ do total de casos registrados e também foi maioria em todos os anos do estudo.

Tabela 1: Casos de tuberculose notificados no Sistema de Informação de Agravos de Notificação (SINAN) e taxa de crescimento negativo no município de Tianguá - CE, no período de 2008 a 2013.

\begin{tabular}{|c|c|c|c|c|c|c|c|c|}
\hline Casos notificados & 2008 & 2009 & 2010 & 2011 & 2012 & 2013 & $\begin{array}{c}\text { TCN } \\
(2008-2013)\end{array}$ & Total \\
\hline & 23 & 16 & 18 & 18 & 12 & 11 & $52,17 \%$ & 98 \\
\hline
\end{tabular}

TcN: taxa de crescimento negativo

Um estudo epidemiológico da TB foi realizado no município de Ribeirão Preto, SP e nele foram encontrados $71,7 \%$ pacientes do gênero masculino e $28,3 \%$ pacientes do gênero feminino (7). Outro trabalho, realizado no município de Manaus, AM, apontou como maioria as notificações de tuberculose de pacientes do gênero masculino, apresentando $59,4 \%$ do total de casos (8). Em Londrina, PR, foi encontrada a predominância da doença em $68,1 \%$ em pacientes do gênero masculino (9).

Ao longo da série histórica, essa predominância de pessoas do gênero masculino infectadas vem se re- petindo. Este aspecto ainda não está bem esclarecido na literatura da área, mas há inferências como uma maior exposição dos homens aos fatores de risco, uma menor taxa de utilização dos serviços de saúde e aspectos culturais ligados aos modos de viver, de ser saudável e de adoecer da população masculina (10).

Na Grande Cidade de Praia, da endêmica Santiago Cabo Verde, o autor verificou uma elevada predominância da tuberculose pulmonar no sexo masculino em comparação com o sexo feminino, concordando com o presente estudo (11).

Tabela 2: Caracterização dos casos de tuberculose notificados no Sistema de Informação de Agravos de Notificação (SINAN) do município de Tianguá, CE, segundo o gênero (2008-2013).

\begin{tabular}{|c|c|c|c|c|c|c|c|c|c|c|c|c|c|c|}
\hline \multirow{2}{*}{ Gênero } & \multicolumn{2}{|c|}{2008} & \multicolumn{2}{|c|}{2009} & \multicolumn{2}{|c|}{2010} & \multicolumn{2}{|c|}{2011} & \multicolumn{2}{|c|}{2012} & \multicolumn{2}{|c|}{2013} & \multicolumn{2}{|c|}{ Total } \\
\hline & $\mathrm{n}$ & $\%$ & $n$ & $\%$ & $n$ & $\%$ & $n$ & $\%$ & $\mathrm{n}$ & $\%$ & $n$ & $\%$ & $\mathrm{n}$ & $\%$ \\
\hline Masculino & 15 & 65,2 & 11 & 68,8 & 10 & 55,6 & 13 & 72,2 & 09 & 75,0 & 06 & 54,5 & 64 & 65,3 \\
\hline Feminino & 08 & 34,8 & 05 & 31,2 & 08 & 44,4 & 05 & 27,8 & 03 & 25,0 & 05 & 45,5 & 34 & 34,7 \\
\hline
\end{tabular}

Fatores como maior exposição a riscos relativos a ambientes de trabalho, alcoolismo e tabagismo, além de retardo na procura de serviços de saúde, são apontados como determinantes da maior prevalência de TB em homens (12).

Em estudo realizado em Belo Horizonte, MG, foi observado um predomínio do sexo masculino, confirmando o perfil observado em estudos não controlados sobre tuberculose (13).

Com relação à escolaridade, foram predominantes os indivíduos que possuíam até o Ensino Fundamental com $57,2 \%$ do total. O índice de analfabetismo foi de $16,4 \%$ conforme consta na Tabela 3 .
Foi observado um número alto de indivíduos com baixa escolaridade infectados $(73,6 \%$ somando analfabetismo e ensino fundamental). Um trabalho realizado no município de Piripiri - PI teve incidência de analfabetismo entre esses indivíduos de 68,4\% (14). A pouca escolaridade parece um fator de inequidade social, associado á doença.

A baixa escolaridade foi identificada como fator de risco independente para desenvolvimento de tuberculose, mais frequente em pacientes com menos de 7 anos de estudo. Estudos anteriores apontaram maior ocorrência de casos em pessoas com baixa escolaridade, condicionante de sua situação de saúde e de seu acesso aos serviços de saúde (15). 


\section{Anozanas}

Os dados de escolaridade encontrados neste estudo foram concordantes com os da literatura, que relata a inexistência ou baixa escolaridade da maioria dos casos, sendo um fator de risco para a tuberculose, contribuindo inclusive para a não adesão ao tratamento e para o aumento da taxa de abandono. A baixa escolaridade da população é reflexo de todo um conjunto de condições socioeconômicas precárias, que aumentam a vulnerabilidade à tuberculose e são responsáveis pela maior incidência da enfermidade e pela menor aderência ao respectivo tratamento (16).

Tabela 3: Caracterização dos casos de tuberculose notificados no Sistema de Informação de Agravos de Notificação (SINAN) do município de Tianguá, CE, segundo a escolaridade (2008-2013).

\begin{tabular}{|c|c|c|c|c|c|c|c|c|c|c|c|c|c|c|}
\hline & \multicolumn{2}{|c|}{2008} & \multicolumn{2}{|c|}{2009} & \multicolumn{2}{|c|}{2010} & \multicolumn{2}{|c|}{2011} & \multicolumn{2}{|c|}{2012} & \multicolumn{2}{|c|}{2013} & \multicolumn{2}{|c|}{ Total } \\
\hline & n & $\%$ & $n$ & $\%$ & $\mathrm{n}$ & $\%$ & $\mathrm{n}$ & $\%$ & $\mathrm{n}$ & $\%$ & n & $\%$ & $\mathrm{n}$ & $\%$ \\
\hline Analfabeto & 04 & 17,4 & 03 & 18,7 & 03 & 6,7 & 02 & 11,1 & 01 & 8,33 & 03 & 27,3 & 16 & 16,4 \\
\hline Ensino fundamental & 16 & 69,6 & 10 & 62,5 & 09 & 0 & 08 & 44,4 & 07 & 58,3 & 06 & 54,5 & 56 & 57,2 \\
\hline Ensino Medio & 03 & 13 & 01 & 6,25 & 02 & 11 & 02 & 11,1 & 0 & 0 & 01 & 9,1 & 09 & 9,1 \\
\hline Ensino superior & 0 & 0 & 0 & 0 & 0 & 0 & 0 & 0 & 01 & 8,33 & 0 & 0 & 01 & 1,0 \\
\hline Ignorado & 0 & 0 & 01 & 6,25 & 0 & 0 & 01 & 5,6 & 03 & 25,0 & 0 & 0 & 05 & 5,1 \\
\hline Não informado & 0 & 0 & 01 & 6,25 & 04 & 2,2 & 05 & 27,8 & 0 & 0 & 01 & 9,1 & 11 & 11,2 \\
\hline
\end{tabular}

A escolaridade baixa dos pacientes reflete um conjunto de determinantes socioeconômicos, que pode aumentar a incidência de TB e ser responsável pelo aumento dos abandonos de tratamento (6).

Esta variável (escolaridade) mostrou ser limitada, em virtude de terem sido observadas 16 notificações com dados ignorados ou em branco.
Quanto à distribuição da faixa etária, foi observada que a mais atingida pela tuberculose esteve entre 40 e 59 anos com $40,8 \%$. A faixa etária menos atingida foi a de $\leq 19$ anos, com apenas $2 \%$ do total (Tabela 4).

Tabela 4: Caracterização dos casos de tuberculose notificados no Sistema de Informação de Agravos de Notificação (SINAN) do município de Tianguá, CE, segundo a faixa etária (2008-2013).

\begin{tabular}{|c|c|c|c|c|c|c|c|c|c|c|c|c|c|c|}
\hline \multirow{2}{*}{ Faixa etária } & \multicolumn{2}{|c|}{2008} & \multicolumn{2}{|c|}{2009} & \multicolumn{2}{|c|}{2010} & \multicolumn{2}{c|}{2011} & \multicolumn{2}{|c|}{2012} & \multicolumn{2}{|c|}{2013} & \multicolumn{3}{c|}{ Total } \\
\hline & $\mathbf{n}$ & $\%$ & $\mathbf{n}$ & $\%$ & $\mathbf{n}$ & $\%$ & $\mathbf{n}$ & $\%$ & $\mathbf{n}$ & $\%$ & $\mathbf{n}$ & $\%$ & $\mathbf{n}$ & $\%$ \\
\hline$\leq 19$ & 0 & 0 & 0 & 0 & 01 & 5,60 & 0 & 0 & 0 & 0 & 01 & 9,1 & 02 & 2,0 \\
\hline $20-39$ & 09 & 39,1 & 01 & 6,25 & 08 & 44,4 & 02 & 11,1 & 04 & 33,3 & 04 & 36,4 & 28 & 28,6 \\
\hline $40-59$ & 10 & 43,5 & 06 & 37,5 & 07 & 38,9 & 09 & 50,0 & 04 & 33,3 & 04 & 36,4 & 40 & 40,8 \\
\hline$\geq 60$ & 04 & 17,4 & 09 & 56,25 & 02 & 11,1 & 07 & 38,9 & 04 & 33,4 & 02 & 18,1 & 28 & 28,6 \\
\hline
\end{tabular}

Foi constatado que a faixa etária mais acometida estava entre 40 a 59 anos (40,8\%). Resultado semelhante foi encontrado por Reis e cols (2013) em um estudo em Belo Horizonte, MG (17). Em outro trabalho feito em João Pessoa, $\mathrm{PB}$, a faixa etária que predominou esteve entre 20 e 39 anos, com 51,8\% (18). Analisando um estudo feito em Campinas, SP, a faixa etária entre 20 e 39 anos também predominou entre o total de casos (19).

A população idosa também tem sido alvo de preocupação para as políticas de controle dessa endemia em nível mundial, devido à possibilidade de infecção por reativação da doença e por decorrência do processo de envelhecimento (20).

Outro fato que apontou o controle inadequado da doença foi o percentual elevado de adultos jovens infectados, indicando a ocorrência de transmissão recente. Esse padrão epidemiológico difere do encontrado em países onde a doença está mais bem controlada e a população idosa é a mais acometida, em decorrência de exposição no passado (21). 


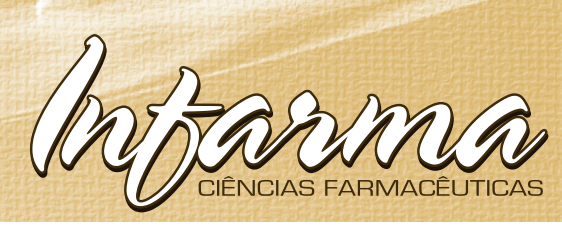

A Tabela 5 mostra os casos de tuberculose notificados no município de Tianguá, CE segundo o tipo de entrada. Durante o período estudado, houve 80 ocorrências consideradas casos novos. Em relação

ao número total de casos, o percentual desse tipo de entrada foi de $81,7 \%$. Os índices de transferência e recidiva foram os mesmos, indicando $6,1 \%$ do total de casos.

Tabela 5: Caracterização dos casos de tuberculose notificados no Sistema de Informação de Agravos de Notificação (SINAN) do município de Tianguá, CE, segundo o tipo de entrada dos pacientes (2008-2013).

\begin{tabular}{|c|c|c|c|c|c|c|c|c|c|c|c|c|c|c|c|}
\hline & \multicolumn{2}{|c|}{2008} & \multicolumn{2}{|c|}{2009} & \multicolumn{2}{|c|}{2010} & \multicolumn{2}{c|}{2011} & \multicolumn{2}{|c|}{2012} & \multicolumn{2}{|c|}{2013} & \multicolumn{2}{c|}{ Total } \\
\hline Entrada & $\mathbf{n}$ & $\%$ & $\mathbf{n}$ & $\%$ & $\mathbf{n}$ & $\%$ & $\mathbf{n}$ & $\%$ & $\mathbf{n}$ & $\%$ & $\mathbf{n}$ & $\%$ & $\mathbf{n}$ & $\%$ \\
\hline Caso novo & 20 & 7,0 & 12 & 75,0 & 11 & 61,1 & 17 & 94,4 & 10 & 83,34 & 10 & 90,9 & 80 & 81,7 \\
\hline Transferência & 02 & 7,0 & 01 & 6,25 & 03 & 16,7 & 0 & 0 & 0 & 0 & 0 & 0 & 06 & 6,1 \\
\hline Recidiva & 01 & 4,3 & 01 & 6,25 & 02 & 11,1 & 01 & 5,6 & 01 & 8,33 & 0 & 0 & 06 & 6,1 \\
\hline Reingresso após abandono & 0 & 0 & 02 & 12,5 & 01 & 5,55 & 0 & 0 & 01 & 8,33 & 01 & 9,1 & 05 & 5,1 \\
\hline Não informado & 0 & 0 & 0 & 0 & 01 & 5,55 & 0 & 0 & 0 & 0 & 0 & 0 & 01 & 1,0 \\
\hline
\end{tabular}

Um estudo realizado no município de Pelotas, RS, em 2008, teve como resultado, em relação ao tipo de entrada, $85,6 \%$ de casos novos (20). Outro estudo, realizado em Campinas, SP, mostrou que houve uma prevalência de casos novos de tuberculose registrados no município, com $82,2 \%$ do total de casos (19).

Os números do Brasil são extremamente preocupantes, seja considerando a situação do país como um todo ou apenas por regiões. Em 2000 foram notificados 82.249 casos novos, sendo 38.690 no sudeste, 23.196 no nordeste, 9.281 no sul, 5.901 no norte e 3.522 no centro-oeste (22).

Foi encontrado ainda um total de $5,1 \%$ de pessoas que voltaram ao tratamento após abandono. Apesar do tratamento da tuberculose ser eficaz e barato, custando entre US\$ 10 e 20 por um período de 6 meses, dependo do país, a taxa de abandono ao tratamento é muito elevada. Isto se deve a duração do tratamento, efeitos colaterais como náuseas, vômitos, icterícia, perda de equilíbrio, asma, alterações visuais, diminuição da audição, neuropatia periférica e até cegueira e a falta de informação e de acompanhamento aos pacientes (1).

Os casos de abandono do tratamento são problemas tão relevantes quanto a própria incidência da doença, visto que é um dos fatores associados ao aumento da falência dos esquemas, devido à resistência do $M$. tuberculosis aos fármacos disponíveis. Também onera o sistema de saúde que deve dispor de recursos humanos e materiais cada vez que o doente de tuberculose inicia um novo tratamento (23).

A Tabela 6 apresenta a distribuição dos casos notificados segundo a forma clínica da TB. As formas clínicas da tuberculose foram agrupadas em três categorias: pulmonar, extrapulmonar e pulmonar + extrapulmonar. A forma pulmonar esteve presente em $85,7 \%$ dos casos; a forma extrapulmonar isolada acometeu $10,2 \%$ dos casos e a associação da forma pulmonar e extrapulmonar representou $4,1 \%$ dos casos.

Tabela 6: Caracterização dos casos de tuberculose notificados no Sistema de Informação de Agravos de Notificação (SINAN) do município de Tianguá, CE, segundo a forma clínica (2008-2013).

\begin{tabular}{|c|c|c|c|c|c|c|c|c|c|c|c|c|c|c|}
\hline \multirow{2}{*}{ Forma clínica } & \multicolumn{2}{|c|}{2008} & \multicolumn{2}{|c|}{2009} & \multicolumn{2}{|c|}{2010} & \multicolumn{2}{|c|}{2011} & \multicolumn{2}{|c|}{2012} & \multicolumn{2}{|c|}{2013} & \multicolumn{3}{|c|}{ Total } \\
\hline & $\mathbf{n}$ & $\%$ & $\mathbf{n}$ & $\%$ & $\mathbf{n}$ & $\%$ & $\mathbf{n}$ & $\%$ & $\mathbf{n}$ & $\%$ & $\mathbf{n}$ & $\%$ & $\mathbf{n}$ & $\%$ \\
\hline Pulmonar & 20 & 87,0 & 14 & 87,5 & 15 & 83,3 & 15 & 83,3 & 09 & 75 & 11 & 100 & 84 & 85,7 \\
\hline Extrapulmonar & 02 & 8,70 & 01 & 6,25 & 03 & 16,7 & 01 & 5,6 & 03 & 25 & 0 & 0 & 10 & 10,2 \\
\hline Ambas as formas clínicas & 01 & 4,30 & 01 & 6,25 & 0 & 0 & 02 & 11,1 & 0 & 0 & 0 & 0 & 04 & 4,1 \\
\hline
\end{tabular}

Em um estudo semelhante, realizado em Chapecó, SC, em 2010, foi avaliada também a forma clínica da tuberculose e foi constatado que a forma pulmonar foi predominante com $77,7 \%$ do total de 225 prontuá- rios e fichas de notificação analisados, seguida das formas extrapulmonares, com $18,66 \%$ e pulmonar + extrapulmonar, com 3,55\% (24). A forma pulmonar é de maior infectividade, portanto os resultados mostram a 
importância do diagnóstico baseado na baciloscopia do escarro para a confirmação e controle adequado dos casos. Essa confirmação da forma pulmonar, o mais precoce possível, favorece a implantação de medidas para a interrupção da cadeia da transmissão da doença (25).

É estimada, na população geral, que cerca de $80 \%$ dos casos de TB sejam da forma pulmonar e $20 \%$ sejam extrapulmonar. Os dados encontrados em nossa série enquadram-se nesta estimativa e, sendo a forma pulmonar da doença a forma infectante, é de se esperar a maior prevalência desses casos, haja vista ser o $M$. tuberculosis uma bactéria aeróbica estrita e os pulmões serem órgãos bastante aerados (15).

A tuberculose pulmonar é a forma clínica da doença de maior acometimento no homem. Foi observado, em um estudo em Porto Alegre, RS, que aproximadamente
$60 \%$ dos pacientes tinham essa forma de apresentação clínica da doença (26). Esses achados reforçam os resultados dessa investigação onde em todos os anos, nos casos de TB, predominou a forma pulmonar (Tabela 6).

A TB pulmonar é um problema mundial de saúde pública, pois o $M$. tuberculosis infecta aproximadamente um terço da população mundial, destacando-se dessa forma como uma das mais significativas causas de morte por doença infecciosa no mundo entre indivíduos adultos, principalmente na faixa etária entre 15 e 49 anos (27).

Estudos realizados em Natal, RN, comprovaram que a TB pulmonar foi a forma mais comum em quatro anos de investigação (27). Segundo os mesmos autores isso se deu pela forma fácil de contágio, pois, até um simples espirro ou gotículas da saliva podem contaminar uma pessoa sadia ou de baixa imunidade.

Tabela 7: Caracterização dos casos de tuberculose notificados no Sistema de Informação de Agravos de Notificação (SINAN) do município de Tianguá, CE, segundo a ocorrência de coinfecção com HIV (2008-2013).

\begin{tabular}{|c|c|c|c|c|c|c|c|c|c|c|c|c|c|c|}
\hline \multirow{2}{*}{ Exame } & \multicolumn{2}{|c|}{2008} & \multicolumn{2}{|c|}{2009} & \multicolumn{2}{|c|}{2010} & \multicolumn{2}{|c|}{2011} & \multicolumn{2}{|c|}{2012} & \multicolumn{2}{|c|}{2013} & \multicolumn{2}{|c|}{ Total } \\
\hline & $n$ & $\%$ & $n$ & $\%$ & $n$ & $\%$ & $n$ & $\%$ & $n$ & $\%$ & $n$ & $\%$ & $n$ & $\%$ \\
\hline Positivo & 0 & 0 & 0 & 0 & 0 & 0 & 01 & 5,6 & 0 & 0 & 0 & 0 & 01 & 1,0 \\
\hline Negativo & 02 & 8,7 & 11 & 68,75 & 18 & 100 & 17 & 94,4 & 11 & 91,7 & 10 & 91 & 69 & 70,4 \\
\hline Em andamento & 12 & 52,2 & 01 & 6,25 & 0 & 0 & 0 & 0 & 01 & 8,3 & 0 & 0 & 14 & 14,3 \\
\hline Não realizado & 09 & 39,1 & 04 & 25,0 & 0 & 0 & 0 & 0 & 0 & 0 & 01 & 9,0 & 14 & 14,3 \\
\hline
\end{tabular}

O desenvolvimento de TB pulmonar resulta da interação de vários fatores: fatores biológicos (idade, sexo, desnutrição, diabetes, vírus da imunodeficiência humana (HIV), fatores comportamentais (tabagismo, alcoolismo) e fatores socioeconômicos (a pobreza, a superlotação e condições precárias de habitação). Alguns destes fatores de risco mais contribuem para infeção, em vez da progressão da doença, ao passo que outros são principalmente responsáveis pela progressão da infeção para a doença (11)

Na Tabela 7, encontra-se a distribuição dos casos segundo coinfecção com HIV. Pode ser notado que houve prevalência de indivíduos com tuberculose não infectados pelo HIV com 70,4\% dos casos notificados, ao passo que os infectados pelo vírus representaram apenas $1 \%$ das ocorrências. A sorologia não foi realizada em 14,3\% dos casos. Nesse momento da análise dos dados foi encontrada uma limitação, pois foram registrados 28 casos, sem possibilidade de diagnóstico para HIV, visto que estavam ainda em andamento ou não haviam sido realizados.

No município de João Pessoa, PB, a sorologia foi negativa em $42 \%$ e positiva em $8,8 \%$ dos pacientes re- gistrados com tuberculose. A sorologia não foi realizada em 38,4\% desses pacientes (18).

Atualmente, um grave problema de saúde mundial é a coinfecção HIV- tuberculose, pois os pacientes HIV positivos são mais propensos à infecção pelo $M$. tuberculosis, devido ao fato de que a infecção depende do estado do sistema imunológico que está completamente debilitado nesses pacientes. Por exemplo, uma pessoa que foi infectada pelo vírus HIV tem 10 vezes mais chances de desenvolver TB do que uma pessoa sadia (1).

Em pesquisa realizada com residentes do Distrito Federal um agravo associado a TB foi o HIV (28). O PNCT (Plano Nacional de Controle da Tuberculose) definiu aqueles que vivem com HIV/AIDS, entre outras, como uma população prioritária, uma vez que estratégias especificas devem ser adotadas para determinados grupos. Isso porque na população em geral a TB vem diminuindo; porém em alguns grupos ela se distribui de forma cada vez menos uniforme e mais concentrada (29). Segundo o Boletim Epidemiológico de 2012, a tuberculose representava a primeira causa de morte em pacientes com AIDS no Brasil. Assim, esses pacientes 


\section{Inotaruma}

têm maior probabilidade de apresentar um desfecho desfavorável ao tratamento da tuberculose (29).

\section{CONCLUSÃO}

O conhecimento do perfil socioeconômico do agravo tuberculose se faz importante não apenas para nortear as políticas públicas, mas também para direcionar as ações de atenção básica, por meio da implementação dessas ações pela Estratégia de Saúde da Família (ESF). Esse estudo de avaliação sobre tuberculose confirmou o que já apontavam outros estudos a respeito do perfil dos pacientes, podendo ser concluido que:

- Houve uma diminuição anual de casos notificados durante o período estudado, com índice de $23,4 \%$ no ano de 2008 e diminuição de quase pela metade de $11,2 \%$ no ano de 2013 .

- O gênero masculino predominou e os casos de tuberculose se concentram na faixa etária dos 40 a 59 anos.

- Com relação à escolaridade, prevaleceram os indivíduos com até Ensino Fundamental. O índice de analfabetismo foi de $16,4 \%$.

- Segundo o tipo de entrada, há uma prevalência de casos novos com percentual de $81,7 \%$.

- A forma clínica mais frequente foi a pulmonar com $85,7 \%$.

- De acordo com a coinfecção com HIV, predominaram os doentes de tuberculose não infectados pelo HIV com $70,4 \%$.
Os resultados obtidos neste trabalho poderão orientar as políticas públicas de saúde direcionadas às reais características da região, contribuindo para um melhor conhecimento e melhor atuação dos profissionais quanto à prevenção e controle da doença.

Com relação aos dados, em sua maioria refletem bem o perfil dos pacientes; entretanto, alguns apresentaram lacunas que poderiam ser sanadas, se todas as fichas fossem preenchidas de forma mais completa pelos profissionais de saúde que notificaram os casos.

A subnotificação/dados incompletos de casos de TB e de informações de encerramentos no SINAN impossibilitam o real conhecimento da situação epidemiológica da TB e prejudicam o planejamento e a avaliação de programas direcionados ao seu controle. Devem ser reforçadas as recomendações a autoridades de saúde relacionadas com o programa de controle da TB nos diversos níveis da federação no sentido de fortalecer a descentralização das ações de controle da tuberculose para Unidades de Saúde (ESF e Unidades Básicas de Saúde), para melhorar a realização de forma efetiva do DOTS, a busca de contatos, a detecção precoce da doença e a oportunidade de tratamento; estabelecer rotina de busca em outros sistemas de informação (Sistema de Informação em Mortalidade e Sistema de Informação Laboratorial de TB) para melhoria da qualidade de informação dos encerramentos de TB; e avaliar o sistema de vigilância da tuberculose no município e nos demais prioritários, a fim de definir as diretrizes e prioridades para melhorar os indicadores de cura, abandono e incidência da doença.

\section{REFERÊNCIAS}

1. Souza MVD. Tuberculose em pacientes HIV-positivos, um grave problema de saúde pública mundial. Rev Bras Farm. 2006; 87 (2): 42-44.

2. Hijjar MA. Controle das doenças endêmicas no Brasil Tuberculose. Rev Soc Bras de Med Trop. 1994; 27 (3): 23-36.

3. BRASIL. Ministério da Saúde. Guia de vigilância epidemiológica. Secretaria de Vigilância em Saúde, Departamento de Vigilância Epidemiológica. 7. ed. Brasília: Ministério da Saúde, 2009. Disponível em: <http://www. saude.gov.br/svs>. Acesso em: agosto de 2015.

4. BRASIL. Ministério da Saúde. Programa Nacional de Controle da Tuberculose. Secretaria de Vigilância em Saúde. Departamento de Vigilância Epidemiológica, Brasília: Ministério da Saúde, 2011. Disponível em: $<$ http://www.saude.gov.br/svs $>$. Acesso em: agosto de 2015 .
5. BRASIL. Ministério da Saúde. Boletim epidemiológico. Coordenação-Geral do Programa Nacional de Controle da Tuberculose. Departamento de Vigilância de Doenças Transmissíveis - DEVIT. Secretaria de Vigilância em Saúde. Brasília: Ministério da Saúde, v. 43, especial tuberculose, mar., 2012. Disponível em: <http://www. saude.gov.br/tuberculose $>$. Acesso em: agosto de 2015 .

6. Pereira, JC, Silva, MR; Costa, RR, Guimarães, MDC, Leite, ICG Rev Saúde Pública. 2015;49:6. Doi:10.1590/ S0034-8910.2015049005304.

7. Hino P, Cunha TN, Villa TCS, Santos CB. Perfil dos casos novos de tuberculose notificados em Ribeirão Preto (SP) no período de 2000 a 2006. Ciên Saúde Coletiva. 2011; 16 (1): 1295-1301.

8. Farias AS. Perfil dos doentes de tuberculose no município de Manaus - Amazonas. (Dissertação de Mestrado). São Paulo: Escola de Enfermagem de Ribeirão Preto da Universidade de São Paulo; 2010. 
9. Costa Júnior WL. Avaliação dos casos de tuberculose notificados no município de Londrina no período de 2001 a 2008. (Dissertação de Mestrado). Londrina: Universidade Estadual de Londrina; 2011.

10. Pinheiro RS, Viacava F, Travassos C, Brito AS. Gênero, morbidade, acesso e utilização de serviços de saúde no Brasil. Ciên Saúde Coletiva. 2002; 7 (4): 687-707.

11. Correio IRB, Correio JLS. Dados epidemiológicos da tuberculose em São Gonçalo do Amarante - RN. Rev Bras Cien Saúde. 2013; 11 (36): 29-37.

12. Furtado JC. A tuberculose pulmonar: caracterização do perfil dos pacientes identificados na cidade da Praia. Grande Cidade da Praia: Universidade Jean Piaget de Cabo Verde, 2013.

13. Mascarenhas MDM, Araújo LM, Gomes KRO. Perfil epidemiológico da tuberculose entre casos notificados no município de Piripiri, Estado do Piauí, Brasil. Rev Epidemiol Serv Saúde. 2005; 14 (1): 7-14.

14. Paixão LMM, Gontijo ED. Perfil de casos de tuberculose notificados e fatores associados ao abandono, Belo Horizonte, MG. Rev Saúde Pública. 2007;41(2):205-13.

15. Barbosa DRM, Almeida MG, Martins LM. Características epidemiológicas, clínicas e espaciais de casos notificados de tuberculose em área hiperendêmica do nordeste do Brasil. Rev Epidemiol Control Infect. 2014; 4 (3): 186-191

16. Reis DCs, Almeida TAC, Quites HFO, Sampaio MM. Perfil epidemiológico da tuberculose no município de Belo Horizonte (MG), no período de 2002 a 2008. Rev Bras Epidemiol. 2013; 16 (3): 592-602.

17. Coêlho DMM, Viana RL, Madeira CA, Cardoso LO. Perfil epidemiológico da tuberculose no Município de Teresina-PI, no período de 1999 a 2005. Epidemiol Serv Saúde. 2010; 19(1):33-42.

18. Coutinho LASA, Oliveira DS, Souza GF, Fernandes Filho GMC, Saraiva MG. Perfil epidemiológico da tuberculose no município de João Pessoa - PB, entre 2007 - 2010. Rev Bras Ciên Saúde. 2012; 16 (1): 35-42.

19. Casellato TFL. Aspectos epidemiológicos da tuberculose no município de Campinas - SP, em 2006. (Dissertação de Mestrado). Campinas: Universidade Estadual de Campinas; 2009.
20. Vasconcellos FCS, Chatkin MN. Perfil epidemiológico da tuberculose em Pelotas - Rio Grande do Sul - Brasil. Rev Bras Ciên Saúde, 2008; 12 (3): 229-238.

21. Ohmori M, Ishikawa N, Yoshiyama T, Uchimura K, Aoki M, Mori T. Current epidemiological trend of tuberculosis in Japan. Int J Tuberc Lung D. 2002; 6(5): 415-23.

22. Hijjar MA, Procopio MJ, Oliveira R, Teixeira GM. A tuberculose no Brasil e no Mundo

23. Bol Pneumol Sanit. 2001; 9 (2).

24. . BRASIL. Ministério da Saúde. Secretaria de Vigilância em Saúde. Guia de Vigilância Epidemiológica. $7^{\mathrm{a}} \mathrm{ed}$. Brasília; 2010.

25. Rosseto DS, Gonçalves PTR. Perfil epidemiológico da tuberculose em Chapecó - SC. (Monografia de Graduação). Santa Catarina: Universidade Comunitária da Região de Chapecó; 2010.

26. Xavier MIN, Barreto ML. Tuberculose na cidade de Salvador, Bahia, Brasil: o perfil na década de 1990. Cad Saúde Pública. 2007; 23 (2): 445-453.

27. Cavalcante EFO, Silva DMGV. Perfil de pessoas acometidas por tuberculose. Rev Rede Enferm Nord. 2013; 14 (4): 720-729.

28. Paiva VS, Pereira M, Moreira JS. Perfil epidemiológico da tuberculose pulmonar em Unidade Sanitária de referência em Porto Alegre, RS. Revista da AMRIGS. 2011; 55 (2): 113-117.

29. Araújo SRL, Pereira ISSD, Brito NOS, Fonseca PCB. Perfil epidemiológico da tuberculose pulmonar na cidade do Natal - RN. J Infect Control. 2015; 4 (1): 16-19.

30. Freire JN. Abandono do tratamento da tuberculose em pacientes residentes no DF em 2012. (TCC Graduação). Brasília: Universidade de Brasília, 2014.

31. BRASIL, Ministério da Saúde. Boletim Epidemiológico. Volume 43 março - 2012. Disponível em < http:// www.saude.rs.gov.br/upload/1337634001_TuberculoseBoletim\%20Epidemio.pdf $>x$ 